\title{
Assessing quality of life using WHOQOL- BREF: a cross-sectional study on the association between quality of life and neighborhood environmental satisfaction, and the mediating effect of health-related behaviors
}

Fiona Y. Wong ${ }^{1}$, Lin Yang ${ }^{2}$, John W. M. Yuen ${ }^{2}$, Katherine K. P. Chang ${ }^{2}$ and Frances K. Y. Wong ${ }^{2 *}$

\begin{abstract}
Background: Quality of life (QOL) is an important component in assessing people's health. Environmental quality can influence people's QOL in the physical health, psychological, social relationships and environment domains. $\mathrm{QOL}$ in the four domains, overall QOL and general heath of residents living in the Kowloon Peninsula of Hong Kong were assessed. The association between satisfaction with the neighborhood environment and QOL, and health-related behaviors which mediated the effect were investigated.

Methods: A sample of 317 residents completed a questionnaire which comprised the WHOQOL-BREF (Hong Kong version) to assess QOL, the International Physical Activity Questionnaire (IPAQ) to study physical activities, and questions on satisfaction with the neighborhood environment, health-related behaviors and socio-demographics. One-way ANOVA and linear regression were used to study the associations between environmental satisfaction and QOL in the four domains, overall QOL and general health, followed by assessing the relationships between environmental satisfaction and the potential health-related behavior mediators with regression tests. Mediation analysis was conducted using multiple linear regressions to study the effects of environmental satisfaction on QOL in the four domains, overall QOL and general health, as well as the potential mediating roles played by various health-related behaviors. A P-value of $<0.05$ was considered as statistically significant.
\end{abstract}

Results: The residents had a relatively higher physical health mean score of $70.83 \pm 12.69$, and a lower environmental mean score of $61.98 \pm 13.76$. Moderate satisfaction with the neighborhood environment had a significant relationship with $\mathrm{QOL}$ in the psychological domain $(\beta=0.170, P=0.006)$, however, this effect was partially mediated by the non-smoking behavior of the residents $(\beta=0.143, P=0.022)$.

\footnotetext{
* Correspondence: frances.wong@polyu.edu.hk

${ }^{2}$ School of Nursing, The Hong Kong Polytechnic University, Hung Hom,

Kowloon, Hong Kong

Full list of author information is available at the end of the article
}

(c) The Author(s). 2018 Open Access This article is distributed under the terms of the Creative Commons Attribution 4.0 International License (http://creativecommons.org/licenses/by/4.0/), which permits unrestricted use, distribution, and reproduction in any medium, provided you give appropriate credit to the original author(s) and the source, provide a link to the Creative Commons license, and indicate if changes were made. The Creative Commons Public Domain Dedication waiver (http://creativecommons.org/publicdomain/zero/1.0/) applies to the data made available in this article, unless otherwise stated. 


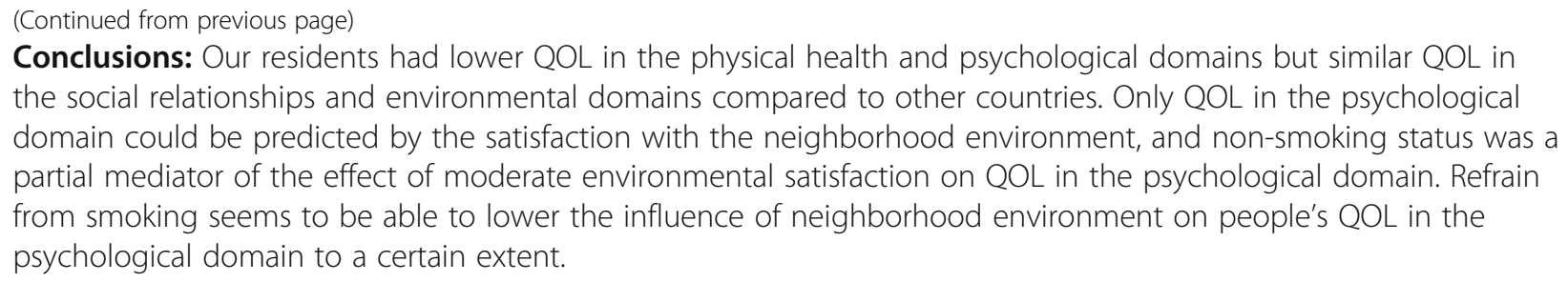

Keywords: Quality of life, Psychological health, Neighborhood environment, Smoking, WHOQOL-BREF

\section{Background}

Quality of life (QOL) is an important component in assessing people's health. It commonly focuses on physical and mental health and functional performance of individuals, however, QOL can be measured in a broad range. The World Health Organization Quality of Life: Brief Version (WHOQOL-BREF) assesses QOL in four domains including physical health, psychological, social relationships and environment [1]. QOL refers to individuals' perception of their position in life in the context of the culture and value systems in which they live and in relation to their goals, expectations, standards and concerns. The QOL in the four domains can be affected by different factors like age, sex, rural and urban areas $[2,3]$, and health and disease status [4].

Concerns for environmental and greening impact on QOL and general health are rising these days. The quality of both the physical and built environment can influence people's perception of general health, well-being and QOL, especially to the youth and elderly [5-7]. Air pollutants and toxins, noise and dirtiness have a negative impact on people's QOL in the physical and psychological domains [8]. Pollution-related diseases, such as respiratory infections, asthma and cardio-pulmonary diseases, could lower people's QOL [9]. Long-term noise exposure not only can cause hearing impairment, but also hypertension, ischemic heart disease, annoyance, sleep disturbance, depression and anxiety [10]. Features of the environment such as accessibility to green space and availability of safe parks, have been found positively associated with mental health and negatively related to stress level $[8,11]$.

Besides environmental factors, QOL can also be affected by health-related behaviors. A study observed that participating in physical activities was associated with better QOL in both the physical and environmental domains [12], however, another study focusing on people with major depressive disorder found that physical activity could only improve QOL in the physical domain [13]. Walking for leisure was also positively associated with QOL in social relationships and environmental domains among men; however, it was positively associated with physical, psychological and environmental domains among women [14]. In addition to physical activities, people who practiced health behaviors like refrain from smoking and following a healthy diet were usually more psychologically healthy with less depressive symptoms $[15,16]$.

Social economic status (SES) including income, education and occupation could have a certain effect on the living environment of an individual. Previous studies found that those who had higher income and education level tended to live in less polluted neighborhood with more access to green and open spaces [10, 17]. The more healthy and green environment usually brings higher QOL especially in the psychological and environmental aspects [5, 7].

Hong Kong is a highly dense city with over 7 million populations, located at the southern coast of China with an area of approximately $1100 \mathrm{~km}^{2}$ [18]. Although around $50 \%$ are woodland and shrub land, most of them are inaccessible. Open space is a luxury rather than a basic necessity. Only $2.3 \%$ of the lands are for parks, stadiums, playgrounds and recreational facilities. Air pollution and excessive noise are also long-lasting issues in Hong Kong because of crowdedness and heavy traffic. A previous study reported that environmental quality was one of the important infrastructures contributed to QOL of the university students in Hong Kong, however, environment and greening were the least satisfactory ones [19]. Since greenery and open spaces are limited in Hong Kong, we aimed to study the effect of satisfaction with the neighborhood environment on residents' QOL, and the potential of encouraging health-related behaviors to improve QOL.

The objectives of this study were (i) to assess the QOL in the four domains, including physical, psychological, social relationships and environmental, and overall QOL and general heath, of residents living in the Kowloon Peninsula of Hong Kong, (ii) to study the effect of satisfaction with the neighborhood environment on QOL, and (iii) to identify health-related behaviors which mediated the effect of satisfaction with the neighborhood environment on QOL.

\section{Methods}

\section{Target population}

Hong Kong consists of three main territories including Hong Kong Island, Kowloon Peninsula and New Territories. 
There are 127 district council constituency areas in the Kowloon Peninsula. Nine district council constituency areas near Hung Hom and Tsim Sha Tsui located in the southwest of Kowloon Peninsula were selected as the target study sites. These areas cover mixed-use commercial and residential districts, old urban and more affluent districts with private and public housing, and people with low and high SES [20]. According to the 2011 Hong Kong Population Census, there were 158,103 people living in these areas [20]. Among this population, $88.0 \%$ were Chinese and $53.8 \%$ were female. Age distribution was comparable to the whole population in Hong Kong. Residents aged 18 years or above and had been living in the nine areas for at least 90 days were the target population. Those who were cognitively impaired or unable to communicate effectively in Cantonese, Mandarin and English were excluded. Assuming that $50 \%$ of the population was satisfied with the environment, a sample size of 266 would provide a precision of $6 \%$ from the true values at $95 \%$ confidence level.

\section{Instrument}

The validated WHOQOL-BREF (Hong Kong version) was used to measure QOL $[1,21]$. It consists of 24 items to assess perception of quality of life in four domains, including physical health, psychological, social relationships and environment, and two items on overall QOL and general health. The domain scores were transformed into a linear scale between 0 and 100 following the scoring guidelines [1]. A higher score indicated a better QOL. Physical activities were assessed by the International Physical Activity Questionnaire short form (IPAQ-SF) which is adequately reliable and valid in a Chinese population [22]. Walking, moderate-intensity and vigorous-intensity activities were assessed. The total MET-minutes/week was calculated for each participant. Metabolic Equivalent of Task (MET) is the energy cost of physical activities [23]. Physical activity levels were categorized as low, moderate and high, based on the criteria listed on the IPAQ guidelines [22]. People have to answer all questions on number of days in a week and daily time performing walking, moderate and vigorous activities in order to calculate their total MET and identify their physical activity levels. Those who were unable or refused to answer all these questions were removed from the analysis according to the IPAQ guidelines [22]. Smoking, alcohol drinking, and vegetable and fruit intakes were also assessed. For practicing low fat, low salt and low sugar diets, respondents could choose "never (1)", "seldom (2)", "sometimes (3)" and "always (4)". Seven questions on satisfaction with the neighborhood environment with a five-point Likert scale (1: very unsatisfied; 5 : very satisfied) were used to evaluate air quality, ventilation, drinking water quality, noise condition, lighting condition, environmental hygiene, and environmental protection and recycling. Six questions on satisfaction with open spaces including greening, parks and gardens, recreation and sports facilities, promenade, rest areas and pedestalization were also assessed using the same Likert scale. Socio-demographic profiles of the residents were also assessed.

Test-retest reliability of the questionnaire had been tested with 64 subjects before implementation of the main study. All question items showed a Cohen's kappa value ranged from $0.50-1.00$ or an intraclass correlation coefficient value ranged from $0.55-1.00$, which indicated that the reliability of the questionnaire was fair to excellent.

\section{Procedures}

This was a cross-sectional study using a convenience sampling method to recruit potential participants. Trained interviewers approached people at parks, resting areas and outside food markets and shopping centers in the nine district areas from 9 am to $7 \mathrm{pm}$. In addition to weekdays, the questionnaire interviews were also conducted on weekends to reach residents who spent most of their time in other districts on weekdays. After confirming the eligibility of participation, the purpose of the questionnaire survey was explained. If the potential participants were in a group, only one family member from each household was allowed to answer the questionnaire. A verbal consent was obtained from each of the participants. The answered questionnaire and the signing for receiving the token of appreciation were also the implied consent to participate in the study. A face-to-face interview was conducted on the recruitment site. A HKD 50 (approximately USD 6.45) supermarket shopping voucher was given as a token of appreciation to those who completed the questionnaire.

\section{Statistical analysis}

Data analysis was performed using SPSS version 21. Descriptive statistics were reported by mean \pm standard deviation or proportion, as appropriate. Satisfaction levels with physical environment and open spaces across different districts were compared using chi-square tests. Relationships between QOL in the four domains, overall QOL and general health were assessed using Spearman's correlations.

Mediation analysis was conducted to study the effects of overall levels of satisfaction with the neighborhood environment (independent variable) on QOL in the four domains, overall QOL and general health (criterion variables), as well as the potential mediating roles played by various health-related behaviors (Fig. 1). To obtain the overall levels of satisfaction with the neighborhood enevironment, the mean scores of those environmental and open spaces variables which showed significant differences across the nine districts were summed to calculate the range of the overall environmental satisfactory 


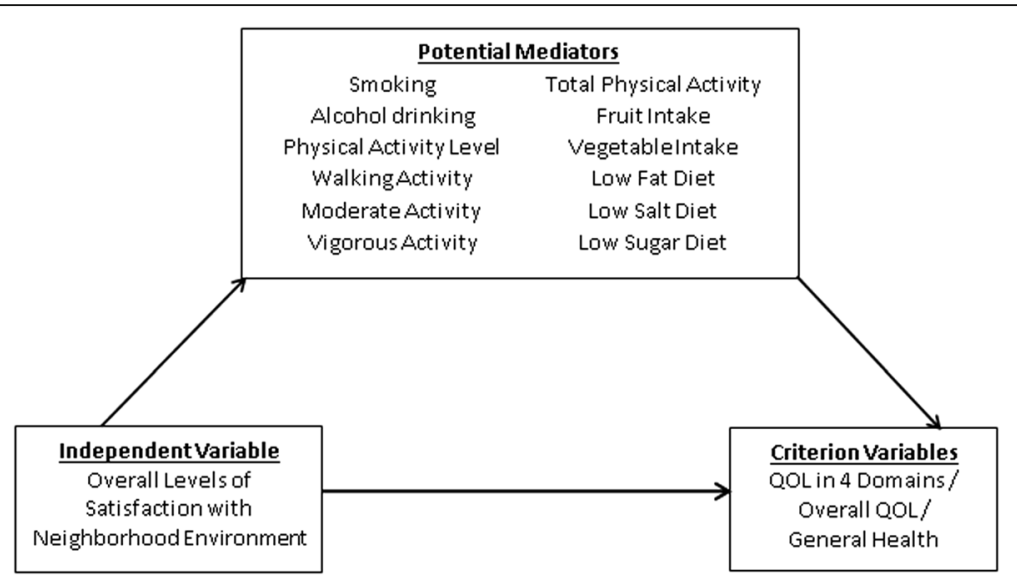

Fig. 1 Hypothetical mediated model

scores (1: very unsatisfied; 5: very satisfied). Scores in the lowest $1 / 3$ were categorized as "low satisfaction"; the second $1 / 3$ were categorized as "moderate satisfaction", while the highest $1 / 3$ were categorized as "high satisfaction". To conduct the mediation analysis, the existence of relationships between the independent variable and criterion variables were assessed using one-way ANOVA and linear regression in the first place, followed by assessing the existence of relationships between the independent variable and the potential health-related behavior mediators with regression tests. Mediated models with the combined effects of overall levels of satisfaction with the neighborhood environment and each of the health-related behaviors in predicting QOL were evaluated using multiple linear regression tests. Regression coefficients were used to assess the effect of mediation and further confirmed by both the Aroian and Goodman tests. A $P$-value of $<0.05$ was considered as statistically significant.

\section{Results}

\section{Socio-demographics}

A total of 317 residents were successfully interviewed during July - September 2015. The majority were Chinese (99\%), 44\% were male, $60 \%$ were married and the mean age was 45.12 years $(\mathrm{SD}=17.28$ ) (Table 1$)$. Nearly $46 \%$ had been living in the nine district areas for more than 10 years and $46 \%$ were living in a self-owned private permanent housing. About 33\% had attained a university level. Around 25\% claimed that they had no income while $31 \%$ received an individual monthly income more than HKD 14800 (USD 1922), the median monthly income of the Hong Kong population in 2014 [24].

\section{Health-related behaviors}

A total of $10.5 \%$ of the residents smoked every day, almost every day or occasionally (Table 1). About $18.3 \%$ claimed that they consumed alcohol at least 2-4 times/ month. Regarding fruit and vegetable intakes, $13.2 \%$ and $7.6 \%$ had achieved the recommended intake of at least 2 servings of fruits and 3 servings of vegetables a day, respectively. More than $60 \%$ claimed that they sometimes or always practiced low fat, low salt or low sugar eating. For physical activity, those who were unable or refused to estimate their number of days or daily time performing walking, moderate and/or vigorous activities were removed from the analysis. 240 out of 317 respondents completed the IPAQ assessment. Most of them were categorized as having moderate physical activity level (62.9\%), and $13.3 \%$ were having low activity level or not performing physical activity.

\section{Satisfaction with environment and open spaces}

The residents were most satisfied with water quality (55.4\%) in their living districts, whereas they were most dissatisfied with air quality (29.7\%), ventilation (27.1\%) and noise level (25.9\%). For open spaces, they were most satisfied with the promenade (36.9\%) but another $22.7 \%$ were dissatisfied with the promenade. On the other hand, $25.2 \%$ and $22.1 \%$ were dissatisfied with the pedestrianization and greening in the open spaces, respectively. Crosstabulations of the living districts and satisfaction with physical environment and open spaces found that satisfaction levels with air quality $(P=0.029)$, noise condition $(P=0.041)$, parks and gardens $(P=$ $0.015)$, recreation and sports facilities $(P=0.034)$ and promenade $(P<0.001)$ were significantly different across the nine districts (Table 2).

\section{Quality of life}

Table 3 shows the scores of QOL in the four domains, overall QOL and general health. Comparing the four domains of the residents, physical health domain was the highest with a mean score of $70.83 \pm 12.69$ while the 
Table 1 Socio-demographic characteristics and health-related behaviors of the residents $(N=317)$

\begin{tabular}{lll}
\hline & $n(\%)$ & Mean \pm SD \\
\hline Sex & \\
Male & $139(43.8 \%)$, \\
Female & $178(56.2 \%)$
\end{tabular}

Age (years)

\begin{tabular}{|c|c|}
\hline $18-24$ & 48 (15.1\%) \\
\hline $25-44$ & 107 (33.8\%) \\
\hline $45-64$ & 115 (36.3\%) \\
\hline$\geq 65$ & 47 (14.8\%) \\
\hline
\end{tabular}

Ethnicity

$\begin{array}{ll}\text { Chinese } & 313(99.1 \%) \\ \text { Non-Chinese } & 3(0.9 \%) \\ \text { Missing } & 1 \\ \text { Length of residence in the district (yrs) } & \\ \leq 5 & 95(30.0 \%) \\ 6-10 & 75(23.7 \%) \\ 11-20 & 64(20.2 \%) \\ \geq 21 & 83(26.2 \%)\end{array}$

Types of housing

Self-owned private permanent housing

Rental private permanent housing

Rental public housing

Others

Missing

Education

Primary school or below

Secondary school

Diploma/Certificate

University degree

$145(45.9 \%)$

$34(10.8 \%)$

$87(27.5 \%)$

$50(15.8 \%)$

1

$61(19.2 \%)$

$117(36.9 \%)$

$33(10.4 \%)$

$106(33.4 \%)$

Individual monthly income (HKD)

$$
\begin{aligned}
& \$ 0 \\
& \$ 10,500 \text { or below } \\
& \$ 10,501-14,800 \\
& \$ 14,801-23,000 \\
& \geq \$ 23,001
\end{aligned}
$$

Marital status

Never married

Married

Widowed/Divorced/Separated

Current smoking status

Never smoke

$284(89.6 \%)$

Occasionally

$10(3.2 \%)$

\begin{tabular}{|c|c|c|}
\hline & $n(\%)$ & Mean $\pm S D$ \\
\hline \multicolumn{3}{|l|}{ Alcohol drinking } \\
\hline Never & 165 (52.1\%) & / \\
\hline 1 or $<1 /$ mth & 94 (29.7\%) & \\
\hline $2-4 / \mathrm{mth}$ & $40(12.6 \%)$ & \\
\hline $2-3 / w k$ & 10 (3.2\%) & \\
\hline$\geq 4 / w k$ & $8(2.5 \%)$ & \\
\hline \multicolumn{3}{|l|}{ Fruit intake } \\
\hline Never & $13(4.1 \%)$ & / \\
\hline$<1$ serving/wk & $173(54.6 \%)$ & \\
\hline 1 serving/day & 89 (28.1\%) & \\
\hline$\geq 2$ servings/day & $42(13.2 \%)$ & \\
\hline
\end{tabular}

$23(7.3 \%)$

Everyday ${ }^{\mathrm{a}} /$ Almost every day
Table 1 Socio-demographic characteristics and health-related behaviors of the residents $(N=317)$ (Continued)

Vegetable intake

Never

$5(1.6 \%)$

$<1$ serving/day

$127(40.1 \%)$

1 serving/day

$92(29.0 \%) \quad /$

2 servings/day

$69(21.8 \%)$

$\geq 3$ servings/day

$24(7.6 \%)$

Practicing low fat diet

Never

$48(15.1 \%) \quad /$

Seldom

$55(17.4 \%)$

Sometimes

$122(38.5 \%)$

Always

$92(29.0 \%)$

Practicing low salt diet

$\begin{array}{ll}\text { Never } & 51(16.1 \%) \\ \text { Seldom } & 60(18.95) \\ \text { Sometimes } & 114(36.0 \%) \\ \text { Always } & 92(29.0 \%)\end{array}$

Practicing low sugar diet

$\begin{array}{ll}\text { Never } & 46(14.5 \%) \\ \text { Seldom } & 66(20.8 \%) \\ \text { Sometimes } & 104(32.8 \%) \\ \text { Always } & 101(31.9 \%)\end{array}$

Physical Activity Level $(N=240)$

Low $32(13.3 \%) \quad$ /

$\begin{array}{ll}\text { Moderate } & 151(62.9 \%) \\ \text { High } & 57(23.8 \%)\end{array}$

Types of Activities (MET-minutes/week)

Walking /

$1343.60 \pm 1075.91$

Moderate-intensity activity

$361.98 \pm 759.74$

Vigorous-intensity activity

$357.92 \pm 739.23$

Total physical activity

$2142.94 \pm 1661.32$

${ }^{a}$ Every day: one stick of cigarette a day or 7 sticks of cigarettes a week for at least 6 months 


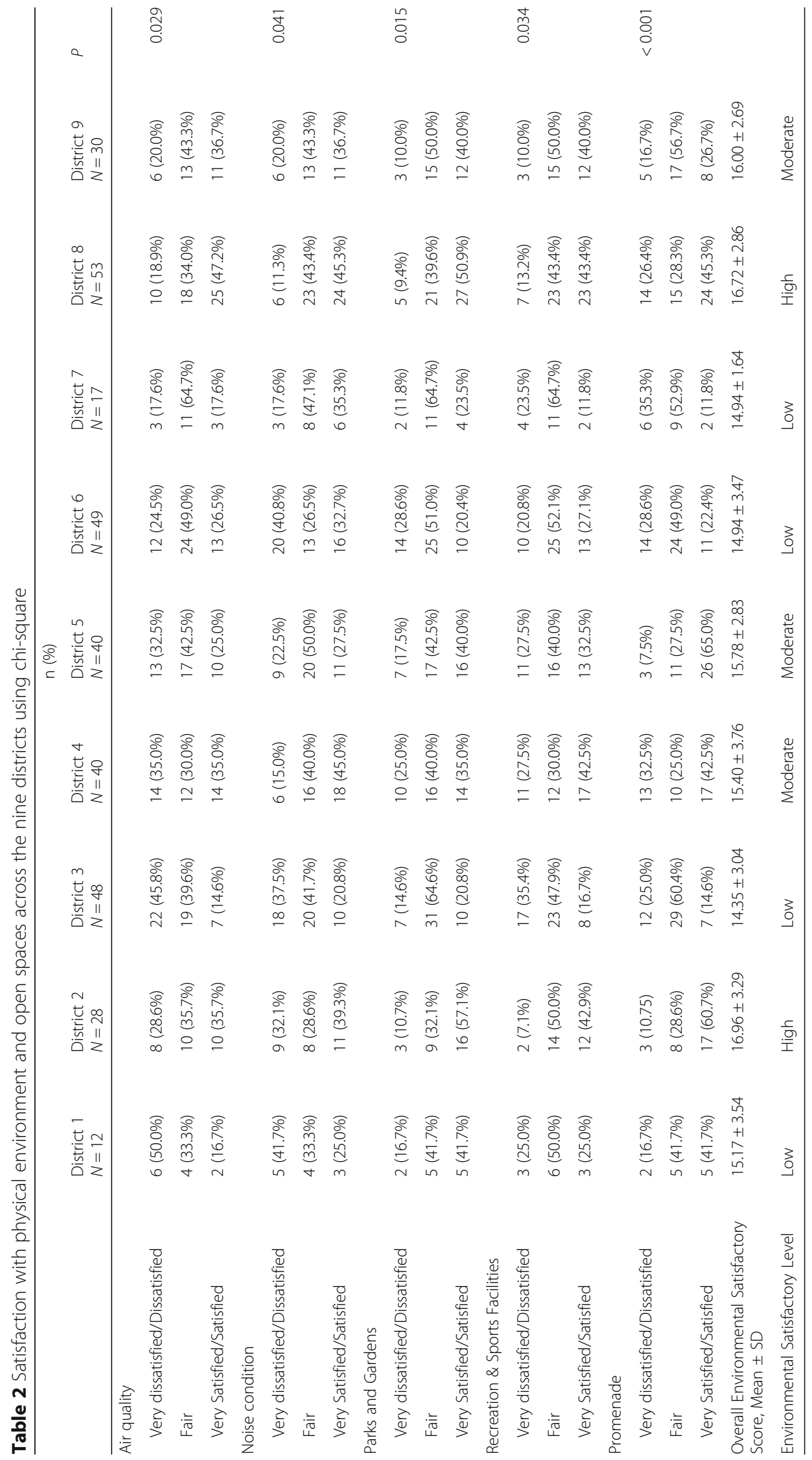


Table 3 Scores of the 4 QOL domains, overall QOL and general health and their spearman's correlations $(N=317)$

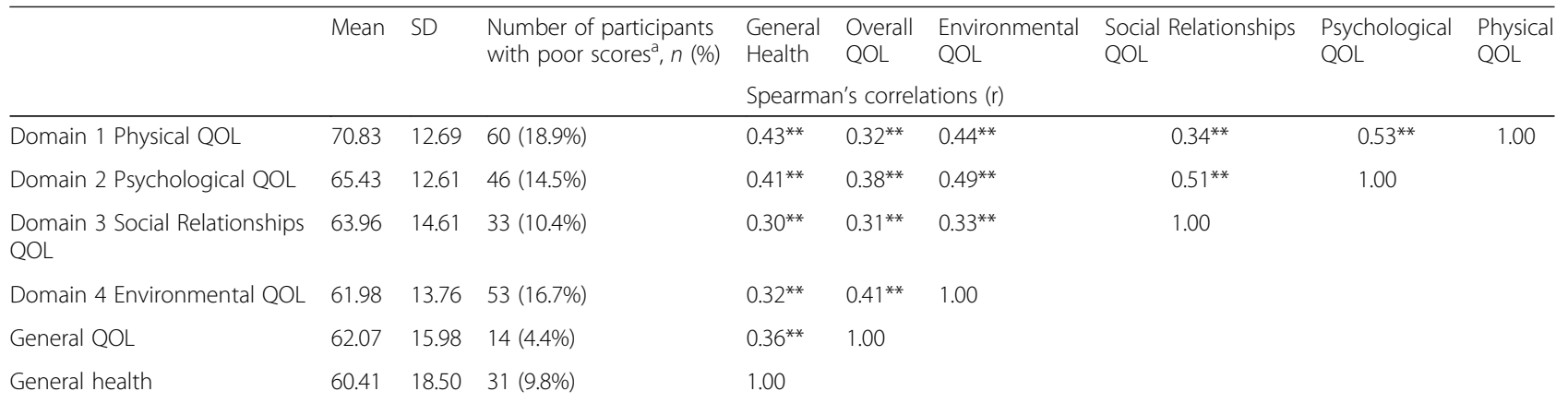

${ }^{a_{\text {scores }}<1 S D}$

$* * P<0.01$

environmental domain was the lowest with a mean score of $61.98 \pm 13.76$. Using one SD below the mean as the cut-off standards for low QOL [23], 18.9\% of the respondents were considered to have poor QOL in the physical domain, followed by environmental domain (16.7\%), psychological domain (14.5\%) and social relationships domain (10.4\%). The four domains, overall QOL and general health were significantly and positively interrelated with low to moderate relationships $(r=0.30-0.53$, $P<0.01$ ) (Table 3).

\section{Mediation analysis \\ Overall levels of satisfaction with the environment}

To calculate the overall environmental satisfactory scores, the mean scores of the physical environment and open spaces variables which showed significant differences across the nine districts including air quality, noise condition, parks and gardens, recreation and sports facilities, and promenade, were summed. The overall environmental satisfactory scores in the lowest $1 / 3(14.35-15.22)$ were categorized as "low satisfaction"; the second 2/3 (15.2316.09) were categorized as "moderate satisfaction", while the highest $1 / 3(16.10-16.96)$ were categorized as "high satisfaction" (Table 2).

\section{Associations between the independent variable and the criterion variables}

One-way ANOVA tests showed that the overall levels of satisfaction with the neighborhood environment only significantly predicted QOL in the psychological domain $(P=0.020)$ (Table 4$)$. Further analysis using linear regression found that residents with moderate satisfaction with the neighborhood environment had significantly higher QOL in the psychological domain $(\beta(95 \% \mathrm{CI})=0.170$ (1.30-7.72), $P=0.006)$. Associations between the overall levels of satisfaction with the environment and the other three QOL domains, overall QOL and general health were insignificant.

\section{Associations between the independent variable and the potential mediators}

Regression analyses showed that overall levels of satisfaction with the neighborhood environment were significantly related to smoking status, alcohol drinking, physical activity level, and walking activity and total physical activity measured in MET-minutes/week $(P<0.05)$ (Table 5$)$. They were the potential mediators which might have an effect with the overall levels of satisfaction with the neighborhood environment in predicting QOL in the psychological domain. Fruit and vegetable intakes, practice of low fat, low salt and low sugar diet eating, and moderate and vigorous activities were not significantly related to the overall levels of satisfaction with the neighborhood environment.

\section{Associations between the potential mediators and the criterion variable}

Each of the potential health-related behaviors identified was tested with the combined effect of overall levels of satisfaction with the neighborhood environment in predicting QOL in the psychological domain. Table 6 showed the results of each of the models.

Referring to the beta coefficients and Pearson's correlations, only non-smoking was significantly correlated with QOL in the psychological domain $(\beta=0.157, P=$ $0.005 ; \quad \mathrm{r}=0.178 ; \quad P=0.001$ ) (Model 1 ). Non-smokers $(64.03 \pm 12.68)$ were found to have significantly higher QOL in the psychological domain than those who smoked occasionally $(61.92 \pm 9.22)$ and every day $(61.74 \pm$ 9.36). In the non-mediated model, moderate satisfaction with the neighborhood environment significantly predicted QOL in the psychological domain $(\beta=0.170, P=0.006)$ (Table 4), however, when mediated by non-smoking status, the beta weight was lowered to $0.143(P=0.022)$ but remained significant (Model 1). This indicated that the effect of moderate satisfaction with the neighborhood environment on QOL in the psychological domain was partially mediated by the non-smoking status of the residents. 


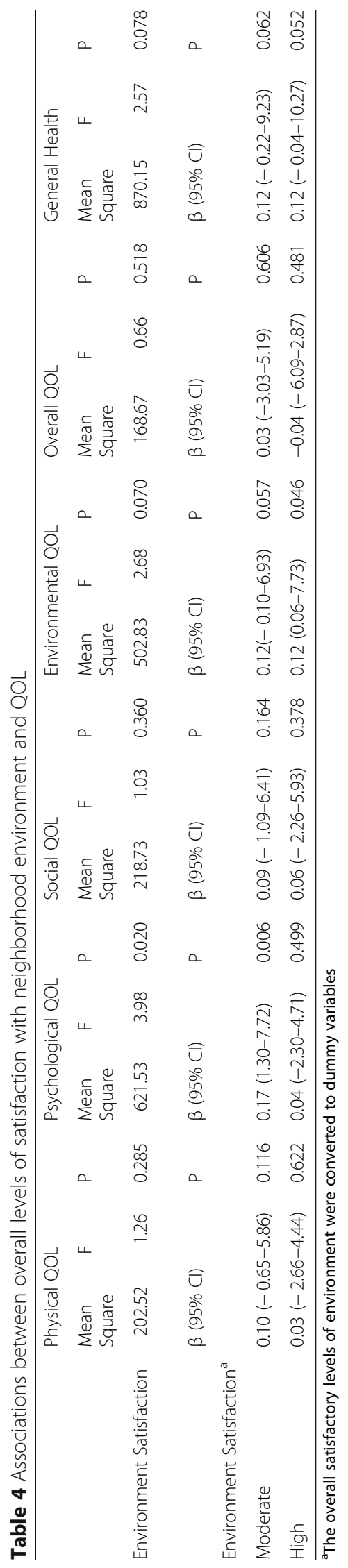


Table 5 Associations between overall levels of satisfaction with neighborhood environment and health-related behaviors

\begin{tabular}{|c|c|c|c|}
\hline & \multicolumn{3}{|c|}{ Environment Satisfaction } \\
\hline & \multicolumn{2}{|l|}{$x^{2}$ a } & $P$ \\
\hline Smoking status & \multicolumn{2}{|l|}{9.97} & 0.007 \\
\hline Alcohol drinking & \multicolumn{2}{|l|}{13.19} & 0.010 \\
\hline Fruit intake & \multicolumn{2}{|l|}{4.95} & 0.084 \\
\hline Vegetable intake & \multicolumn{2}{|l|}{4.84} & 0.089 \\
\hline Low fat diet & \multicolumn{2}{|l|}{3.76} & 0.709 \\
\hline Low salt diet & \multicolumn{2}{|l|}{4.00} & 0.677 \\
\hline Low sugar diet & \multicolumn{2}{|l|}{4.02} & 0.674 \\
\hline \multirow[t]{2}{*}{ Physical activity level } & \multicolumn{2}{|l|}{12.04} & 0.017 \\
\hline & Mean Square ${ }^{b}$ & $\mathrm{~F}$ & $P$ \\
\hline Walking activity & $55,503,381$ & 4.89 & 0.008 \\
\hline Moderate activity & $794,945.73$ & 1.38 & 0.253 \\
\hline Vigorous activity & $1,149,574.02$ & 2.12 & 0.122 \\
\hline Total physical activity & $12,137,648.59$ & 4.53 & 0.012 \\
\hline
\end{tabular}

${ }^{a}$ Multinominal regression test

${ }^{\mathrm{b}}$ Linear regression test

Other health-related behaviors including alcohol drinking, physical activity levels, walking activity and total physical activity were not significantly related to QOL in the psychological domain (Models 2-5).

\section{Quantifying the partial mediation effect}

The statistical significance of the mediation effect was further confirmed by both the Aroian test $(Z=1.964$, $\mathrm{SE}=0.375, P=0.049)$ and the Goodman test $(\mathrm{Z}=2.088$, $\mathrm{SE}=0.353, P=0.037)$. The beta coefficient of the effect of moderate satisfaction with the neighborhood environment on non-smoking status $(\beta=0.178, P=0.004)$ (not shown), and the beta coefficient of the effect of non-smoking status on QOL in the psychological domain $(\beta=0.157, P=0.005)$, gave a product of beta coefficient of $0.0279(0.178 \times 0.157)$ in the mediated path. The indirect effect of 0.0279 and the total direct effect of 0.170 gave a ratio of 0.1641 , which indicated that $16.41 \%$ of the effect of moderate satisfaction with the neighborhood environment on QOL in the psychological domain was mediated through the non-smoking status (Fig. 2). Part of the relationship between moderate satisfaction with neighborhood environment and QOL in the psychological domain was due to the effect of non-smoking status on the psychological domain.

\section{Discussion}

Among the four domains of QOL, the residents in this study had a relatively higher QOL in the physical health domain and a lower QOL in the environmental domain. An overall moderate satisfaction with the neighborhood environment was found significantly predicted QOL in
Table 6 Effects of overall levels of satisfaction with neighborhood environment and health-related behaviors in predicting psychological QOL

\begin{tabular}{|c|c|c|c|c|}
\hline & \multicolumn{4}{|c|}{ Psychological QOL } \\
\hline & \multicolumn{2}{|c|}{$\begin{array}{l}\text { Multiple Linear } \\
\text { Regression }\end{array}$} & \multicolumn{2}{|c|}{$\begin{array}{l}\text { Pearson's } \\
\text { Correlation }\end{array}$} \\
\hline & $\bar{\beta}$ & $P$ & $r$ & $P$ \\
\hline \multicolumn{5}{|l|}{ Model 1} \\
\hline \multicolumn{5}{|c|}{ Environment Satisfactory levels* } \\
\hline Moderate & 0.143 & 0.022 & 0.153 & 0.003 \\
\hline High & 0.036 & 0.560 & -0.031 & 0.291 \\
\hline \multicolumn{5}{|l|}{ Smoking status* } \\
\hline Non-smoking & 0.157 & 0.005 & 0.178 & 0.001 \\
\hline \multicolumn{5}{|l|}{ Model 2} \\
\hline \multicolumn{5}{|c|}{ Environment Satisfactory levels* } \\
\hline Moderate & 0.180 & 0.004 & 0.153 & 0.003 \\
\hline High & 0.042 & 0.498 & -0.031 & 0.291 \\
\hline \multicolumn{5}{|l|}{ Alcohol drinking* } \\
\hline Never & 0.070 & 0.575 & 0.021 & 0.356 \\
\hline Occasionally & 0.024 & 0.849 & -0.020 & 0.364 \\
\hline
\end{tabular}

Model 3

Environment Satisfactory levels*

$\begin{array}{lllll}\text { Moderate } & 0.133 & 0.071 & 0.135 & 0.019\end{array}$

$\begin{array}{lllll}\text { High } & 0.006 & 0.937 & -0.049 & 0.224\end{array}$

Physical activity levels*

$\begin{array}{lllll}\text { High } & 0.180 & 0.056 & 0.080 & 0.108 \\ \text { Moderate } & 0.143 & 0.128 & 0.012 & 0.427\end{array}$

Model 4

Environment Satisfactory levels*

$\begin{array}{lllll}\text { Moderate } & 0.170 & 0.014 & 0.146 & 0.009 \\ \text { High } & 0.030 & 0.669 & -0.030 & 0.315 \\ \text { Walking activity } & 0.105 & 0.090 & 0.095 & 0.061\end{array}$

Model 5

Environment Satisfactory levels*

\begin{tabular}{lllll} 
Moderate & 0.133 & 0.071 & 0.135 & 0.019 \\
High & -0.003 & 0.964 & -0.049 & 0.224 \\
Total physical activity & 0.103 & 0.117 & 0.103 & 0.056 \\
\hline
\end{tabular}

"The overall satisfactory levels of environment, smoking status, alcohol drinking, and physical activity levels were converted to dummy variables

the psychological domain, however, this effect was partially mediated by the non-smoking status of the residents.

This study covered both where the affluent and the relatively poor people were living. According to the census statistics, the demographic characteristics of our respondents were comparable to the whole population in Hong Kong in terms of sex and age [25, 26]. However, our respondents were more educated, mostly married and received lower monthly income. 
Non-mediated

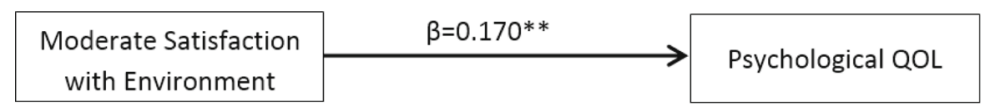

Mediated

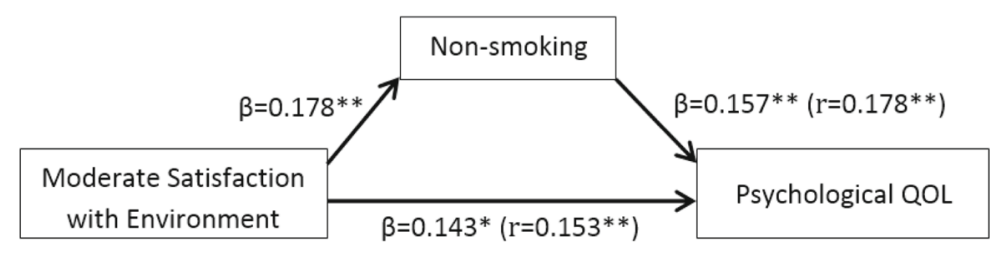

$* \mathrm{P}<0.05$

$* * P<0.01$

Fig. 2 The non-mediated and mediated models with path coefficients. ${ }^{*} P<0.05$, ${ }^{*} P<0.01$

Our residents showed lower QOL in the physical health and psychological domains compared with the mean scores reported by the 23 countries in the WHOQOL Group but QOL in the social relationships and environmental domains were comparable [27]. When comparing with another healthy sample in Hong Kong [20], our residents had slightly lower QOL in the physical health domain (score difference: - 0.48) but better QOL in the psychological (score difference: +6.49 ), social relationships (score difference: +2.52 ) and environmental domains (score difference: +1.92 ). Our sample also showed better QOL scores in all domains compared with another Chinese population living in an urban community in China [26]. Using one SD below the mean as the cut-off standards for low QOL, however, a higher proportion in our study had poor QOL in all the four domains compared with this Chinese population [26].

Significant correlations between QOL in the four domains, overall QOL and general health were observed. Our findings are consistent with previous studies which showed that the four domains of QOL and perceived general health are interrelated. People with positive emotions or better QOL in the psychological domain evidence better physical health outcomes, such as fewer physical complaints, more exercise, longer sleeping hours and better sleep quality [28]. Increasing transient emotions can strengthen immune functioning and buffer the impact of stress which gives people better health $[28,29]$. On the other hand, exposure to nature or green space has been found to improve people's health and well-being by providing restoration from stress and mental fatigue [30]. In areas where $90 \%$ of the environment around the home was green, 5.3\% less residents would feel unhealthy compared with areas in which only $10 \%$ of the environment was green [5]. Detrimental social relationships also play a role in physical and psychological health. An adverse family environment and lack of social support may result in depressive symptoms and subsequent psychological distress which in turn would affect one's general health [31]. To facilitate social interactions and networking, a neighborhood with better built environment, such as street connectivity, traffic and pedestrian safety, improved air quality and greenery are necessary.

Studies have shown that people tend to have better mental health if they are living in an environment which is less affected by noise and increasing temperatures, with better air quality, plenty of vegetation and open spaces, adequate social and entertainment facilities, and safe to go out in the day and at night [31-33]. Our study found that there were significant differences in levels of satisfaction with air quality, noise pollution, parks and gardens, recreation and sports facilities, and promenade across the nine districts. The results also supported that residents who were moderately satisfied with the neighborhood environment had significantly higher QOL in the psychological domain compared with those who had low levels of satisfaction, however, the effect stopped at moderate satisfaction level. High levels of satisfaction with the neighborhood environment could not further raise QOL in the psychological domain.

The availability and accessibility of open spaces and leisure facilities, and the quality of air and noise condition could play an important role in the overall levels of satisfaction with the environment. Residents living in Districts 4 and 5 were moderately satisfied with the environment. Green and open spaces including a garden, a rest-area and a promenade are located in both districts. A relatively higher proportion of residents in District 4 were satisfied with the noise condition, and recreation \& sports facilities while residents in District 5 were more satisfied with the promenade. Districts 8 and 9 are 
residential areas with lower SES but they are far from the busiest streets and traffic. The highest proportion of people satisfied with air quality, noise condition, recreation and sports facilities were found in District 8. This may explain why residents living in Districts 8 and 9 were highly and moderately satisfied with the environment, respectively. Districts 1, 3, 6 and 7 were the four districts with the lowest overall satisfaction with the environment. Main streets are running across Districts 1 and 6. Residents were most dissatisfied with air quality and noise pollution. Open spaces, parks and recreational facilities are obviously inadequate, or difficult to access in these districts. District 3 is close to the waterfront, nevertheless, residents were obviously dissatisfied with the air and noise qualities and the recreational and sports facilities nearby. Quality of green and open spaces are sometimes more crucial compared with quantity [34]. To understand the views of the residents in depth, interviews or focus group discussions are needed. The existing outdoor and recreational facilities may not be able to meet the needs of the residents, or they need to be renovated.

The sole effect of overall satisfaction with the neighborhood environment on QOL in the psychological domain can be explained by its potential influence on sleeping quality, green exercise, social contacts and cohesion. Green spaces help filtering pollution from the air and reduce air and ground temperature, which facilitate people to achieve a healthier duration of sleep. People do not sleep well have been observed to have adverse mood and cognitive performance. Moreover, increasing temperature has been associated with more aggressive behaviors and higher suicide rate, and stress-related disorders [35]. People who are living in a less crowded place or presence in a more natural environment would have more opportunities to involve activities in green places. Green exercise significantly decreases stressful events, loneliness and depression, while better QOL, happiness and social interaction are promoted $[6,11,35]$. A neighborhood with better built environment such as walkability and street connectivity can improve people's mental health and well-being by increasing their social cohesion [29, 34, 36].

We found that potential health-related behaviors mediators including smoking status, alcohol drinking, physical activity levels, walking activity and total physical activity were significantly correlated with the overall satisfaction with the neighborhood environment. The quality of the neighborhood residential environment or neighborhood physical disorder has been found to be related to some of the health damaging behaviors such as cigarette smoking, alcohol and drugs [37, 38]. Neighborhood physical disorders are usually observed in areas with violence, poor safety at night, street littering, and traffic and neighborhood noise which could lead to psychological distress [39].
The high smoking rate in neighborhoods with physical disorders could be explained by the fact that there are fewer options for pleasurable activities, hence smoking is seen as one of the few pleasurable activities [38]. Other studies explained that disorderly and unsafe environments attract men whose SES is subordinate [40, 41]. The act of smoking can show a certain type of masculinity. In Hong Kong, however, due to land limitation and high housing price, most of the time mid-to high SES groups have to live in neighborhoods with undesirable environmental quality which they are not really satisfied with. More local studies are needed to investigate the associations of smoking, alcohol and environment, and compare with the western studies.

A systematic review showed that neighborhood environment had an association with walking for exercise while lack of equipment and facilities were only significantly related to sports and exercise activity [42]. These results are consistent with our findings which found that overall environmental satisfaction was significantly correlated with walking activity only, but not moderate and vigorous physical activities. Regarding walking activity, the supportiveness of neighborhood environmental attributes is crucial $[36,42]$. People tend to walk more often if the air quality is less polluted, facilities are aesthetics, parks are safe, and street are well-connected. Moderate and vigorous physical activities are usually sports, endurance or strengthening exercises which require equipment and facilities. People can look for appropriate facilities out of their living districts, therefore, the influence of living environment is reduced.

In regard to the associations of health-related behaviors and QOL in the psychological domain, smoking status was the only health-related behaviors observed in this study. In previous studies, moderate alcohol drinking, however, was found positively associated with mental health because of the possibilities of social factors connected with alcohol [43]. Moderate-to-vigorous physical activity had also been reported to be positively correlated with psychological health-related QOL [44], but it has no relationship with QOL in the psychological domain in this study. Relationships between socio-demographic characteristics, such as age and sex, and QOL were also studied in previous studies, however, the findings were inconsistent. A study found that women aged $57-70$ years exhibited significantly higher QOL in the physical, social relationships and environmental domains than men in the same age group, but nearly identical QOL in the psychological domain [45]. Another study found that women had significantly lower QOL in the psychological and social relationships domains possibly because women trended towards more depressed than men [46]. In future studies, relationships between socio-demographic factors, QOL and 
satisfaction with neighborhood environment can be considered.

The mediation analysis showed that the relationship of moderate satisfaction with the neighborhood environment and QOL in the psychological domain was partially mediated $(16.41 \%)$ if the residents were non-smokers since the non-smoking status also significantly increased QOL in the psychological domain. QOL deteriorates with an increase in daily cigarette smoked [16, 47]. Non-smokers usually have higher QOL in the physical, mental and social functioning domains. Because of nicotine on neurotransmitter activity in the brain, smokers have been reported to have more depressive symptoms, as well as more complaints of weakness, headache, dizziness, pain and discomfort [16]. Smoking and depression are part of the same vicious cycle. Without any stress coping skills, they would smoke when they are stressed or depressed. The relief of the feelings of irritability and anxiety claimed by smokers could be simply the nicotine withdrawal effect after they have not smoked for a while. In many societies smoking is a discouraged or an unacceptable behavior causes some smokers to feel stressful and uneasy when smoking in public [48]. Same in Hong Kong, smoking is banned in many of the public indoor and outdoor areas. This combination of physical and mental influences could lower smokers' QOL in the psychological domain. In other words, non-smokers usually have better QOL in the psychological domain. Other studies also identified that residential satisfaction and sense of community (i.e. the feeling that one is part of a larger dependable and stable structure) could mediate the impact of environment on psychological well-being, life satisfaction and positive affect $[7,49]$.

\section{Limitations}

A convenience sampling method was used in this study and residents who participated were mainly those who stayed outdoors or used the facilities and amenities in the neighborhood. Those who spent most of their time indoors or at home were less likely to be approached. These limited our studying of the QOL of residents and their perspectives of the environment. A random sampling telephone interview could be an alternative in the future. The low specificity but high sensitivity of the IPAQ-SF and the potential of inaccuracy of self-reported data could also lead to the insignificant mediating effect of physical activity levels, walking activity and total physical activity observed on the relationship of satisfaction with neighborhood environment and QOL in the psychological domain. Individual in-depth interviews or focus group discussions together with questionnaire survey can help data triangulation to facilitate understanding of the reasons behind the ratings of the environment and open spaces. In future studies, objective data such as vegetation density, air pollutants and traffic noise should be considered to supplement the self-rated environmental data. Other factors like residential satisfaction, sense of community, and family and neighbor relationships which may potentially mediate the effect of neighborhood environment on QOL can be further investigated.

\section{Conclusions}

This study assessed the QOL of residents living in the Kowloon peninsula of Hong Kong and the effect of overall satisfaction with the neighborhood environment on QOL. Our residents had lower QOL in the physical health and psychological domains but similar QOL in the social relationships and environmental domains compared to other countries. As the sample of residents were from different districts in Kowloon, their overall satisfaction with environment in the neighborhood varied. A significant relationship between satisfaction with the neighborhood environment and QOL in the psychological domain was found. The non-smoking status of the residents partially mediated the effect of moderate environmental satisfaction on the psychological domain. In a dense city like Hong Kong where green and open spaces are limited, refrain from smoking seems to be able to lower the influence of neighborhood environment on people's QOL in the psychological domain to a certain extent. Health promotion and smoking cessation programs can stress the importance of not smoking in enhancing QOL in the psychological domain. This study also provides policy makers and health administrators with evidence-based information on how physical and built environment can influence residents' QOL especially in the psychological domain as well as facilitating the development of environmental interventions and policy recommendations.

\section{Abbreviations}

IPAQ-SF: International Physical Activity Questionnaire- Short Form; MET: Metabolic Equivalent of Task; QOL: Quality of life; SES: Social Economic Status; WHOQOL-BREF: World Health Organization Quality of Life: Brief Version

\section{Acknowledgements \\ We would like to thank the residents who participated in the questionnaire survey and all the interviewers for data collection. \\ Funding \\ This study was funded by Central Funding of The Hong Kong Polytechnic University and Dean's Reserve of Faculty of Health and Social Sciences. \\ Availability of data and materials \\ The datasets generated and/or analyzed during the current study are not publicly available due to participants' consent but are available from the corresponding author via collaboration request.}

\section{Authors' contributions}

FKYW led the research. FYW, LY and FKYW designed the study. FYW wrote the first and subsequent drafts of the manuscript and conducted the statistical analyses. FYW, LY, JWMY, KKPC and FKYW involved in interpretation of the data and revising the manuscripts. All authors have read and approved the 
final version of the manuscript and agreed to be accountable for all aspects of the work.

\section{Ethics approval and consent to participate}

All procedures performed in studies involving human participants were in accordance with the ethical standards of the institutional and/or national research committee and with the 1964 Helsinki declaration and its later amendments or comparable ethical standards. The study protocol was approved by the Human Subjects Ethics Sub-committee (HSESC) of The Hong Kong Polytechnic University (Reference Nos: HSEARS20150121008-01). A verbal informed consent was obtained from each of the participants. The answered questionnaire and the signing for receiving the token of appreciation were also the implied consent to participate and provide data. The above arrangement was approved by the HSESC of The Hong Kong Polytechnic University. Names of the participants, first four digits of their Hong Kong identity cards, and dates providing verbal consent and receiving the token of appreciation were recorded.

\section{Consent for publication}

Not applicable.

\section{Competing interests}

The authors declare that they have no competing interests.

\section{Publisher's Note}

Springer Nature remains neutral with regard to jurisdictional claims in published maps and institutional affiliations.

\section{Author details}

${ }^{1}$ School of Optometry, The Hong Kong Polytechnic University, Hung Hom, Kowloon, Hong Kong. ${ }^{2}$ School of Nursing, The Hong Kong Polytechnic University, Hung Hom, Kowloon, Hong Kong.

\section{Received: 7 December 2017 Accepted: 8 August 2018}

\section{Published online: 12 September 2018}

\section{References}

1. World Health Organization (1996) WHO-BREF: Introduction, administration, scoring and generic version of the assessment. 1996. Geneva: WHO. http:// www.who.int/mental_health/media/en/76.pdf. Accessed 9 August 2017.

2. Apidechkul T. Comparison of quality of life and mental health among elderly people in rural and suburban areas, Thailand. Southeast Asian J Trop Med Public Health. 2011;42:1282-92.

3. Carta MG, Aguglia E, Caraci F, Dell'Osso L, Di Sciascio G, Drago F, et al. Quality of life and urban/rural living: preliminary results of a community survey in Italy. Clin Pract Epidemiol Ment Health. 2012;8:169-74.

4. Bonomi AE, Patrick DL, Bushnell DM, Martin M. Validation of the United States' version of the world health. Organization quality of life (WHOQOL) instrument. J Clin Epidemiol. 2000;53:1-12.

5. Maas J, Verheij RA, Groenewegen PP, de Vries S, Spreeuwenberg P. Green space, urbanity, and health: how strong is the relation? J Epidemiol Community Health. 2006;60:587-92.

6. Van den Berg AE, Maas J, Verheij RA, Groenewegen PP. Green space as a buffer between stressful life events and health. Soc Sci Med. 2010;70: 1203-10.

7. Zhang Z, Zhang J. Perceived residential environment of neighborhood and subjective well-being among the elderly in China: a mediating role of sense of community. J Environ Psychol. 2017:51:82-94.

8. Parra DC, Gomez LF, Sarmineto OL, Buchner D, Brownson R, Schimd T, et al. Perceived and objective neighborhood environment attributes and health related quality of life among the elderly in Bogota, Colombia. Soc Sci Med. 2010;70:1070-6.

9. Darçın M. Association between air quality and quality of life. Environ Sci Pollut Res. 2014;21:1954-9.

10. Lakes T, Bruckner M, Kramer A. Development of an environmental justice index to determine socio-economic disparities of noise pollution and green space in residential areas in berlin. J Environ Plann Man. 2014;57:538-56.

11. Stigsdotter UK, Ekholm O, Schipperijn J, Toftager M, Kamper-Jorgensen F, Randrup TB. Health promoting outdooe environments - associations between green space, and health, health-related quality of life and stress based on a Danish national representative survey. Scand J Public Healt. 2010;38:411-7.

12. Da Silva AT, Coredeiro RC, Ramos LR. Factors associated to quality of life in active elderly. Rev Saúde Pública. 2009;43:613-21.

13. Carta MG, Hardoy MC, Pilu A, Sorba M, Floris AL, Mannu FA, et al. Improving physical quality of life with group physical activity in the adjunctive treatment of major depressive disorder. Clin Pract Epidemiol Ment Health. 2008;4:1.

14. Pucci G, Reis RS, Rech CR, Hallal PC. Quality of life and physical activity among adults: population-based study in Brazilian adults. Qual Life Res. 2013;21:1537-43.

15. Jacka FN, Kremer PJ, Berk M, de Silva-Sanigorski AM, Moodie M, Leslie ER, et al. A prospective study of diet quality and mental health in adolescents. PLoS One. 2011;6:e24805.

16. Taylor G. Change in mental health after smoking cessation: systematic review and meta-analysis. BMJ. 2014;348:g1151.

17. Science for Environment Policy. Links between noise and air pollution and socioeconomic status. In-depth Report 13 produced for the European Commission, DG Environment by the Science Communication Unit, UWE, Bristol. 2016. http://ec.europa.eu/environment/integration/research/ newsalert/pdf/air_noise_pollution_socioeconomic_status_links_IR13_en.pdf. Accessed 4 July 2018.

18. Planning Department. Land utilization in Hong Kong 2017. Planning Department, The Government of HKSAR https://www.pland.gov.hk/pland en/info_serv/statistic/landu.html. Accessed 31 July 2018.

19. $\mathrm{Ng} \mathrm{SL}$. Subjective residential environment and its implications for quality of life among university students in Hong Kong. Soc Indic Res. 2005;71:467-89.

20. Census and Population Department, HKSAR. 2011 Population Census, District Profiles. http://www.census2011.gov.hk/en/constituency-area.html. Accessed 9 August 2017.

21. Leung KF, Wong WW, Tay MSM, Chu MML, Ng SSW. Development and validation of the interview version of the Hong Kong Chinese WHOQOLBREF. Qual Life Res. 2005;14:1413-9.

22. IPAQ (2005) Guidelines for Data Processing and Analysis of the International Physical Activity Questionnaire (IPAQ) - Short and Long Forms. November 2005. https://sites.google.com/site/theipaq/scoring-protocol. Accessed 9 August 2017.

23. Jetté M, Sidney K, Blümchen G. Metabolic equivalents (METS) in exercise testing, exercise prescription, and evaluation of functional capacity. Clin Cardinol. 1990;13:555-65.

24. Census and Statistics Department, HK SAR (2015) 2014 report on annual earnings and hours survey. March 2015 https://www.statistics.gov.hk/pub/ B10500142014AN14B0100.pdf Accessed 9 August 2017.

25. Census and Statistics Department, HKSAR. Hong Kong Annual Digest of Statistics, 2016 edition. https://www.statistics.gov.hk/pub/ B10100032016AN16B0100.pdf Accessed 9 August 2017.

26. Xia P, Li N, Hau KT, Liu C, Lu Y. Quality of life of Chinese urban community residents: a psychometric study of the mainland Chinese version of the WHOOOL-BREF. BMC Med Res Methodol. 2012:12:37.

27. Skevington SM, Lotfy M, O'Connell KA. The World Health Organization's WHOQOL-BREF quality of life assessment: psychometric properties and results of the international field trial. A report from the WHOQOL group. Qual Life Res. 2004:13:299-310.

28. Howell RT, Kern ML, Lyubomirsky S. Health benefits: meta-analytically determining the impact of well-being on objective health outcomes. Health Psychol Rev. 2007;1:83-36.

29. Kok BE, Coffey KA, Cohn MA, Catalino LI, Vacharkulksemsuk T, Algoe SB, et al. How positive emotions build physical health: perceived positive social connections account for the upward spiral between positive emotions and vagal tone. Psychol Sci. 2013;24:1123-32.

30. Groenewegen PP, van den Berg AE, de Vries S, Verheij RA. Vitamin G: effects of green space on health, well-being, and social safety. BMC Public Health. 2006;6:149.

31. Fuhrer R, Stansfeld SA, Chemali J, Shipley MJ. Gender, social relations and mental health: prospective findings from an occupational cohort (Whitehall II study). Soc Sci Med. 1999:48:77-87.

32. Berry HL, Bowen K. Climate change and mental health: a causal pathways framework. Int J Public Health. 2010;55:123-32.

33. Honold J, Beyer R, Lakes T, van der Meer E. Multiple environment burdens and neighborhood- related health of city residents. J Environ Psychol. 2012:32:305-17. 
34. De Vries S, van Dillen SME, Groenewegen PP, Spreeuwenberg P. Streetscape greenery and health: stress, social cohesion and physical activity as mediators. Soc Sci Med. 2013;94:26-33.

35. Barton J, Pretty J. What is the best dose of nature and green exercise for improving mental health? A multi-study analysis. Environ Sci Technol. 2010; 44:3947-55.

36. Renalds A, Smith TH, Hale PJ. A systematic review of built environment and health. Fam Community Health. 2010;33:68-78.

37. Wang X, Auchincloss AH, Barber S, Mayne SL, Griswold ME, Sims M, Diez Roux AV. Neighborhood social environment as risk factors to health behavior among African Americans: the Jackson heart study. Health Place. 2017;45:199-207.

38. Latkin CA, Kennedy RD, Davey-Rothwell MA, Tseng TY, Czaplicki L, Baddela A, Edwards C, Chander G, Moran MB, Knowlton AR. The relationship between neighborhood disorder and barriers to cessation in a sample of impoverished inner-city smokers in Baltimore- Maryland, United States. Nicotine Tob Res. 2017:1-6.

39. Mayne SL, Auchincloss AH, Moore KA, Michael YL, Tabb LP, Echeverria SE, et al. Cross-sectional and longitudinal associations of neighborhood social environment and smoking behavior: the multiethnic study of atherosclerosis. J Epidemiol Commun H. 2017;71:396-403.

40. Miles R. Neighborhood disorder and smoking: findings of a European urban survey. Soc Sci Med. 2006;63:2464-75.

41. Ross CE. Walking, exercising and smoking: does neighborhood matter? Soc Sci Med. 2000;51:265-74.

42. Humpel N, Owen N, Leslie E. Environmental factors associated with adults' participation in physical activity. Am J Prev Med. 2002;22:188-99.

43. Gonzalez-Rubio E, San Mauro I, Lopez-Ruiz C, Díaz-Prieto LE, Marcos A, Nova E. Relationship of moderate alcohol intake and type of beverage with health behaviors and quality of life in elderly subjects. Qual Life Res. 2016; 24:1931-42

44. Buder I, Zick C, Waitzman N. Health-related quality of life associated with physical activity: new estimates by gender and race and ethnicity. World Medical and Health Policy. 2016;8:409-20.

45. Kirchengast S, Haslinger B. Gender differences in health-related quality of life among healthy aged and old-aged Australians: cross-sectional analysis. Gend Med. 2008:5(3):270-8.

46. Bonsaksen T. Exploring gender differences in quality of life. Mental Health Rev J. 2012;17(1):39-49.

47. Goldenberg M, Danovitch I, IsHak WW. Quality of life and smoking. Am J Addict. 2014;23:540-62.

48. Stuber J, Galea S, Link BG. Smoking and the emergence of a stigmatized social status. Soc Sci Med. 2008;67:420-30.

49. Philips DR, Siu O, Yeh AGO, Cheng KHC. The impacts of dwelling conditions on older persons' psychological well-being in Hong Kong: the mediating role of residential satisfaction. Soc Sci Med. 2005;60:2785-97.

Ready to submit your research? Choose BMC and benefit from:

- fast, convenient online submission

- thorough peer review by experienced researchers in your field

- rapid publication on acceptance

- support for research data, including large and complex data types

- gold Open Access which fosters wider collaboration and increased citations

- maximum visibility for your research: over $100 \mathrm{M}$ website views per year

At BMC, research is always in progress.

Learn more biomedcentral.com/submissions 